\title{
Quantum revivals, geometric phases and circle map recurrences
}

\author{
S. Seshadri, S. Lakshmibala and V. Balakrishnan \\ Department of Physics, Indian Institute of Technology - Madras, \\ Chennai 600 036, India
}

\begin{abstract}
Revivals of the coherent states of a deformed, adiabatically and cyclically varying oscillator Hamiltonian are examined. The revival time distribution is exactly that of Poincaré recurrences for a rotation map: only three distinct revival times can occur, with specified weights. A link is thus established between quantum revivals and recurrences in a coarse-grained discrete-time dynamical system.
\end{abstract}

PACS Nos. 42.50.-p, $42.50 \mathrm{Dv}, 03.65 \mathrm{Sq}$

Keywords: coherent state, wavepacket revival, geometric phase, circle map, Poincaré recurrence 
Revivals of a non-stationary state of a Hamiltonian may be regarded as the quantum analogs of recurrences in a classical dynamical system. In general, when a quantum mechanical system is in a state $|\psi\rangle$, the autocorrelation function $|\langle\psi(0) \mid \psi(t)\rangle|^{2}$ decays from its initial value of unity with increasing time $t$. Under very special circumstances, however, it may return to its initial value at certain instants of time, signalling a full revival. Typically, however, any return would be to a value in a small neighborhood of unity [1], which we shall term a "near revival". This would be the counterpart of a Poincaré recurrence [2] in a classical system, although, of course, there is no direct connection between the revivals of quantum states and recurrences of the classical phase trajectories of the same physical system. Taking into account finite experimental resolution, it is evident that near revivals rather than exact revivals are the readily identifiable phenomena of interest. Revivals and fractional revivals of wavepackets are of considerable current interest in many contexts: atom optics [3], propagation of coherent light through a Kerr medium [4], Rydberg states [5], interacting atom-radiation (Jaynes-Cummings) systems [1, 6, 7] with single and multi-photon coherent and squeezed coherent states, quantum dynamics of boson systems and spin systems [8], and so on.

In this paper, we show that one can indeed establish a close relationship between the near revivals of a wavepacket and recurrences in a discrete-time dynamical system, by essentially mapping the former problem onto the latter. This leads to a convenient and systematic way of analyzing the statistics of revivals. In order to discretize time in an unambiguous and natural way, the parameters in the Hamiltonian are varied adiabatically and cyclically, with a time period $T$. Consequently, it is only at instants separated by an interval $T$ that the Hamiltonian returns to its original self. It is therefore meaningful to look for revivals of a state only at the instants $T, 2 T, \cdots$. In turn, this permits us to analyze the problem in terms of recurrences in a discrete-time map.

As a by-product of the cyclic variation of the parameters in the Hamiltonian, the state vector may pick up an extra non-integrable ("geometric") phase [9]. One may expect the revival times to be affected as a consequence - a priori, to get shifted by the time required to cover the angular excess (or decrement) representing the anholonomy in the semiclassical limit, namely, the corresponding Hannay angle 10, 11]. This is indeed the case [12, and the specific system we work with yields an explicit verification of this result as well. For definiteness, we consider a unitarily deformed oscillator Hamiltonian whose eigenstates are squeezed generalized coherent states, while the spectrum remains linear (equi-spaced). Coherent states and their generalizations [13] are convenient in this regard, as they approximate classical states of radiation. They also provide a setting in which anholonomies (non-integrable phases) can be measured experimentally [14, 15] and 
analyzed theoretically [16, 17], in a physical range extending from the extreme quantum regime to the semiclassical limit. Although revivals, per se, are of greatest physical interest in the case of spectra that are nonlinear (in the quantum numbers), here we restrict our attention to a linear spectrum. (In this case exact revivals correspond, as we shall see, to a trivial periodicity.) Complications such as fractional revivals 18 do not arise here, and this helps us establish in a clear manner a very interesting link between near revivals, anholonomies and recurrences - manifestations, respectively, of quantum interference, non-trivial topology in parameter space, and ergodicity in a classical dynamical system.

We recall that the coherent state $|\zeta\rangle(\zeta \in \mathbb{C})$ of the standard oscillator Hamiltonian $H=\hbar \omega\left(a^{\dagger} a+1 / 2\right)$ is given by $|\zeta\rangle=D(\zeta)|0\rangle$ where $|0\rangle$ is the ground state of $H$, and $D(\zeta)$ is the displacement operator $\exp \left(\zeta a^{\dagger}-\zeta^{*} a\right)$. For each value of $\zeta,|\zeta\rangle$ is represented in the position basis by a Gaussian wavepacket which does not spread with time (under evolution governed by $H$ ). The centre of the wavepacket oscillates according to the classical laws of motion. Using the expansion of $|\zeta(0)\rangle \equiv|\zeta\rangle$ in terms of the eigenstates $\{|n\rangle\}$ of $H$, its correlation function is easily found to be

$$
|\langle\zeta(0) \mid \zeta(t)\rangle|^{2}=\exp \left[2|\zeta|^{2}(\cos \omega t-1)\right] .
$$

Thus, in this linear case, revivals of the initial wavepacket simply amount to periodicity with period $2 \pi / \omega$. To induce a geometric phase, however, we need a Hamiltonian that has more than just a single parameter (analogous to $\omega$ in $H$ ) that can be varied. Further, in order to have a non-vanishing anholonomy (Hannay angle) in the semiclassical limit, the geometric phase must have a dependence on the quantum number $n$ labelling the states [10]. Both these objectives are achieved if we begin with a deformation of $H$ produced by squeezing. For the sake of generality, we include a possible displacement as well, and define the transformed Hamiltonian

$$
\widetilde{H}(\alpha, \beta)=S(\beta) D(\alpha) H D^{\dagger}(\alpha) S^{\dagger}(\beta)
$$

where $\alpha, \beta \in \mathbb{C}$, and $S(\beta)$ is the squeezing operator [19 $S(\beta)=\exp \left(\left(\beta a^{\dagger^{2}}-\beta^{*} a^{2}\right) / 2\right)$. As $D(\alpha)$ and $S(\beta)$ are unitary, $H$ and $\widetilde{H}$ are unitarily equivalent and isospectral. $\widetilde{H}$ has eigenstates $|n, \alpha, \beta\rangle=S(\beta) D(\alpha)|n\rangle(n=0,1, \cdots)$, connected by ladder operators $\tilde{a}$ and $\tilde{a}^{\dagger}$, where $\tilde{a}=S D a D^{\dagger} S^{\dagger}$ and $\left[\tilde{a}, \tilde{a}^{\dagger}\right]=1$.

Under a cyclic, adiabatic variation of the displacement and squeezing parameters $\alpha=$ $\alpha_{1}+i \alpha_{2}$ and $\beta=\beta_{1}+i \beta_{2}$, the state $|n, \alpha, \beta\rangle$ acquires a Berry phase $\gamma_{n}$ that is a sum of contributions from the displacement and squeezing parameters, respectively. The contribution from the variation of the displacement parameter $\alpha$ can be found from the group multiplication law for the elements $\{D(\alpha)\}$ of the Heisenberg-Weyl group, namely,

$$
D(\alpha) D\left(\alpha^{\prime}\right)=D\left(\alpha+\alpha^{\prime}\right) \exp \left[\mathrm{i} \chi\left(\alpha, \alpha^{\prime}\right)\right]
$$


where $\chi\left(\alpha, \alpha^{\prime}\right)$ is twice the area of the triangle with vertices at $0, \alpha^{\prime}$ and $\alpha+\alpha^{\prime}$ in the complex plane. This contribution is independent of $n$ essentially because $\left[a, a^{\dagger}\right]$ is just the identity operator. On the other hand, the contribution from the variation of the squeezing parameter $\beta$ is more involved. The squeezing operator $S(\beta)$ represents an element of the group $S U(1,1)$, with generators $K_{+}=a^{\dagger^{2}} / 2, K_{-}=a^{2} / 2$ and $K_{0}=\left[K_{-}, K_{+}\right] / 2=$ $\left(a a^{\dagger}+a^{\dagger} a\right) / 4$. The multiplication rule is of the form [13]

$$
S(\beta) S\left(\beta^{\prime}\right)=S\left(\beta^{\prime \prime}\right) \exp \left[\mathrm{i} \phi\left(\beta, \beta^{\prime}\right) K_{0}\right]
$$

where $\beta^{\prime \prime}$ is a certain function of $\beta$ and $\beta^{\prime}$. The phase $\phi\left(\beta, \beta^{\prime}\right)$ is related to the area of a certain geodesic triangle on the upper sheet of the hyperboloid $\vec{x} \cdot \vec{x} \equiv x_{0}^{2}-x_{1}^{2}-x_{2}^{2}=1$, where (setting $\arg \beta=\varphi) \vec{x}=(\cosh 2|\beta|,-\sinh 2|\beta| \cos \varphi, \sinh 2|\beta| \sin \varphi)$, on which the parameters of $S U(1,1)$ live. The geometric phase is essentially the solid angle subtended at the origin $\vec{x}=0$ by the invariant area enclosed on the hyperboloid [20]. Since $\vec{x} \cdot \vec{x}=1$, this is just the surface integral over $\left(d x_{1} d x_{2}\right) / x_{0}$ on the hyperboloid. In terms of $\beta$ this integral is

$$
\mathcal{B}=\int d^{2} \beta \frac{\sinh 2|\beta|}{|\beta|}
$$

the integration running over the area enclosed by the loop traversed in the $\beta$-plane in a cyclic variation of the squeezing parameters. Further, owing to the presence of the diagonal generator $K_{0}$ in Eq. (4), as opposed to the unit operator in the case of $D(\alpha)$, there is an additional factor $\left(n+\frac{1}{2}\right)$ in the geometric phase. Collecting these results, we arrive at the expression

$$
\gamma_{n}=-2 \mathcal{A}-\left(n+\frac{1}{2}\right) \mathcal{B}
$$

where $\mathcal{A}$ is the area of the loop traversed in the $\alpha$-plane in one cyclic variation of $\alpha_{1}$ and $\alpha_{2}$. The linear dependence of $\gamma_{n}$ on $n$ [17] implies that $\mathcal{A}$ and $\mathcal{B}$ are determined by $\gamma_{0}$ and $\gamma_{1}$ (and vice versa): $\mathcal{A}=\left(\gamma_{1}-3 \gamma_{0}\right) / 4, \mathcal{B}=\gamma_{0}-\gamma_{1}$.

We now construct the coherent state $|z\rangle$ in the tilde-basis, i.e., $\tilde{a}|z\rangle=z|z\rangle \quad(z \in \mathbb{C})$, so that

$$
|z\rangle=\exp \left(-\frac{|z|^{2}}{2}\right) \sum_{n=0}^{\infty} \frac{z^{n}}{\sqrt{n !}}|n, \alpha, \beta\rangle
$$

In the present instance, an equivalent way of obtaining $|z\rangle$ is by operating on the ground state $|0, \alpha, \beta\rangle$ by the displacement operator $\exp \left(z \tilde{a}^{\dagger}-z^{*} \tilde{a}\right)$. Consider time evolution governed by the Hamiltonian $\widetilde{H}$, while $\widetilde{H}$ itself changes adiabatically owing to a cyclic variation of the parameters $\alpha$ and $\beta$ with a time period $T>>\omega^{-1}$. An initial state $|z(0)\rangle$ 
$\equiv|z\rangle$ (given by Eq. (7)) evolves at time $T$ to

$$
|z(T)\rangle=\exp \left(-\frac{|z|^{2}}{2}\right) \sum_{n=0}^{\infty} \frac{z^{n}}{\sqrt{n !}} \exp \left[-i\left(\gamma_{n}-\left(n+\frac{1}{2}\right) \omega T\right)\right]|n, \alpha, \beta\rangle
$$

Using the results quoted in Eqs. (5) and (6) for $\gamma_{n}$, the correlation function now works out to

$$
|\langle z(0) \mid z(T)\rangle|^{2}=\exp \left[2|z|^{2}(\cos (\omega T+\mathcal{B})-1)\right]
$$

Therefore revivals occur at times $T, 2 T, \cdots$ provided $\omega T+\mathcal{B}=2 \pi p$, where $p=0,1, \cdots-$ that is, provided the cyclic variation of the parameters in the Hamiltonian is carried out in a time period equal to one of the values $(2 \pi-\mathcal{B}) / \omega,(4 \pi-\mathcal{B}) / \omega, \cdots$. This is to be compared with the original revival times $2 \pi p / \omega$ (cf. Eq. (1)). As $-\partial \gamma_{n} / \partial n=\mathcal{B}\left(=\gamma_{0}-\gamma_{1}\right.$ in the present instance) is just the Hannay angle [10, we have here an explicit verification of the staggering of the revival times by precisely the time required to cover this angular excess (or decrement, depending on its sign) [12].

Next, we note that there is a convenient and natural way to describe the evolution of the initial state $|z(0)\rangle$ to the state at times $T, 2 T, \cdots$. Equation (8) can be re-written as

$$
|z(T)\rangle=\exp \left(i\left(\gamma_{0}-\frac{1}{2} \omega T\right)\right)\left|z e^{-i(\omega T+\mathcal{B})}\right\rangle .
$$

Moreover, as the cyclic variation of $\alpha$ and $\beta$ is continued, the state $|n, \alpha, \beta\rangle$ picks up the same additional phase $\gamma_{n}$ in each cycle - i.e., the same Hannay angle $\mathcal{B}$ is added in each interval $T$. Therefore, if $\theta_{k}$ denotes the phase of the complex eigenvalue labelling $|z(k T)\rangle(k=0,1,2, \cdots)$, the evolution is equivalent to a rotation map [21] on a circle $\left(S^{1}\right)$, namely,

$$
\theta_{k+1}=\theta_{k}-2 \pi \Delta(\bmod 2 \pi)
$$

where $2 \pi \Delta=\omega T+\mathcal{B}$. If $\Delta$ is an integer, every value of $\theta$ is periodic, with period 1. This is the case already discussed following Eq. (9). A more general possibility is $\Delta=p / q$, a rational number. Again, every orbit of the map is periodic, but with a period $q$. Correspondingly, every initial state $|z(0)\rangle$ has revivals at times $q T, 2 q T, \cdots$. But rational values of $\Delta$ constitute a set of measure zero. The generic case corresponds to an irrational value of $\Delta$, and this is also the most interesting case. (It is also the most pertinent one from a practical point of view, as it relates directly to near revivals, as we shall see.) As is well known, the map no longer has any periodic orbits, but the iterates of any $\theta_{0}$ cover $S^{1}$ densely as $k \rightarrow \infty$. (Regarded as the Poincaré section for motion on a 2-torus, this is the quasi-periodic case). The dynamics is ergodic but not mixing, with 
a uniform invariant density $\rho(\theta)=1 /(2 \pi)$. Although we no longer have exact revivals in principle, the system comes arbitrarily close to these in practice (near revivals) - precisely the analog of Poincaré recurrences. As a consequence, the following complete analysis of the statistics of near revivals becomes possible.

Consider a prescribed small angular interval $I_{\epsilon}$ of size $2 \pi \epsilon$, located symetrically about the initial phase $\theta_{0}$ on $S^{1}$. Let $\Delta \equiv[\Delta]+\delta$, where $[\Delta]$ stands for the integer defined by $\Delta-1<[\Delta]<\Delta$ (for either $\operatorname{sign}$ of $\Delta$ ), so that $\delta$ is an irrational number satisfying $0<\delta<1$. It is then easy to see that $\theta_{k} \in I_{\epsilon} \Rightarrow\{k \delta\}<\epsilon$, where $\{x\}$ denotes the fractional part of $x$. The corresponding correlation function then merely differs from unity by a term of order $\epsilon^{2}$, because

$$
\begin{aligned}
|\langle z(0) \mid z(k T)\rangle|^{2} & =\exp \left[2|z|^{2}(\cos 2 \pi k \Delta-1)\right] \\
& >\exp \left(4|z|^{2} \pi^{2}\{k \delta\}^{2}\right)>1-4|z|^{2} \pi^{2} \epsilon^{2}
\end{aligned}
$$

To order $\epsilon$, therefore, we may regard a return of $\theta_{k}$ to $I_{\epsilon}$ as a (near) revival. The statistics of the occurrence times of such revivals is then identical to that of the recurrences to an angular interval of size $2 \pi \epsilon$ in the rotation map (11). The solution to the latter problem is given by certain gap theorems for interval exchange transformations [22, 23]. Applying these to the case at hand, we obtain the following results in the long-time limit, after the transients due to specific initial conditions have died out and the invariant measure is attained.

Ergodicity implies that the mean recurrence time (here, the mean time between successive near revivals) is, in units of $T$, the reciprocal of the invariant measure of $I_{\epsilon}$. As this measure is uniform on the circle, the mean recurrence time is just $T / \epsilon$, as one may expect. The distribution of recurrence times is, however, quite remarkable [24]. In general (i.e., for arbitrary $\epsilon$ and $\delta$ ), it is concentrated at no more than three points $T_{1}=k_{1} T, T_{2}=k_{2} T$ and $T_{3}=T_{1}+T_{2}$, where $k_{1}$ and $k_{2}$ are the least positive integers such that

$$
\left\{k_{1} \delta\right\}<\epsilon \text { and } 1-\left\{k_{2} \delta\right\}<\epsilon
$$

respectively. (Recall that $\epsilon \ll 1$; as long as $\epsilon<1 / 2, k_{1} \neq k_{2}$.) It is easy to show that $\epsilon \leq\left\{k_{1} \delta\right\}+1-\left\{k_{2} \delta\right\}$ : when the equality sign applies, only two recurrence times $\left(T_{1}\right.$ and $\left.T_{2}\right)$ occur. If $F(k T)$ denotes the normalized invariant (i.e., post-transient) probability that the revival time is $k T$ ( $k=$ positive integer $)$, then

$$
\begin{gathered}
F(k T)=(1 / \epsilon)\left[\left(\epsilon-\left\{k_{1} \delta\right\}\right) \delta_{k, k_{1}}+\left(\epsilon-1+\left\{k_{2} \delta\right\}\right) \delta_{k, k_{2}}\right. \\
\left.+\left(\left\{k_{1} \delta\right\}+1-\left\{k_{2} \delta\right\}-\epsilon\right) \delta_{k, k_{1}+k_{2}}\right]
\end{gathered}
$$


This is consistent with the requirement $\langle k\rangle=1 / \epsilon$ (which follows from ergodicity) provided $k_{2}\left\{k_{1} \delta\right\}+k_{1}\left(1-\left\{k_{2} \delta\right\}\right)=1$, a relationship that can be established independently. With slight modifications, these results for near revivals continue to hold good in the periodic case $(\delta=$ rational number) as well. They are therefore generic, and do not require any fine-tuning of the parameters in the problem $(\omega, T$ and $\mathcal{B})$.

Revivals are a manifestation of the interference arising from the different phases acquired under time evolution by the stationary basis states in the expansion of a nonstationary state. Hence it is the quantum-number-dependent part of the phase (both dynamical and geometric) that is relevant in this regard. That is why, in the present instance, it is only the coefficient $\mathcal{B}$ in the expression for $\gamma_{n}$ (see Eq. (6)) that plays a role, and not the quantity $\mathcal{A}$. (We have already seen why varying $\alpha$ does not lead to an $n$-dependent geometric phase, while varying $\beta$ does so.) Therefore, as far as revivals are concerned, we may hold $\alpha$ fixed at the value zero throughout (i.e., displacement may be dispensed with altogether), without altering any of the conclusions. A cyclic variation of the squeezing parameters $\beta_{1}$ and $\beta_{2}$ suffices to produce the relevant non-trivial anholonomy. For any given values of $\omega T(\gg 1)$ and $\mathcal{B}$, the near revivals of the correlation function concerned have been shown to be essentially equivalent to the Poincaré recurrences in a rotation map on $S^{1}$. The steady-state distribution of recurrence times is explicitly determined. It is restricted to just three possible values. Their locations and relative frequencies of occurrence are easily found for any given $\delta$ and prescribed $\epsilon$.

As already stated, we have considered near revivals in the case of a linear spectrum. In the general nonlinear case (including, in the quantum optical context, multiphoton coherent states [25]), additional interesting features appear, that have to be taken into account in establishing a mapping between near revivals (allowing for the effects of possible non-integrable phases) and Poincaré recurrences. These include fractional revivals [26] and nonlinear dependence of the geometric phase on the quantum numbers. Details of this investigation will be reported elsewhere.

SS acknowledges financial support from the Council of Scientific and Industrial Research, India in the form of a Senior Research Fellowship. SL thanks the Department of Science and Technology, India, for partial support under the grant SP/S2/E-03/96. We are grateful to S. Govindarajan for helpful discussions and to the referees for their suggestions. 


\section{References}

[1] J. H. Eberly, N. B. Narozhny, and J. J. Sanchez-Mondragon, Phys. Rev. Lett. 44 (1980) 1323.

[2] M. Kac, Probability and Related Topics in Physical Sciences (Interscience, New York, 1959).

[3] G. Rempe, H. Walther, and N. Klein, Phys. Rev. Lett. 58 (1987) 353.

[4] K. Tara, G. S. Agarwal, and S. Chaturvedi, Phys. Rev. A 47 (1993) 5024.

[5] J. Parker and C. R. Stroud, Jr., Phys. Rev. Lett. 56 (1986) 716; L. Marmet, H. Held, G. Raithel, J. A. Yeazell, and H. Walther, ibid. 72 (1994) 3779; J. Wals, H. H. Fielding, J. F. Christian, L. C. Snoek, W. J. van der Zande, and H. B. van Linden van den Heuvell, ibid. 72 (1994) 3783; R. Bluhm, V. A. Kostelecky, and B. Tudose, Phys. Rev. A 52 (1996) 2234 and references therein.

[6] M. V. Satyanarayana, P. Rice, R. Vyas, and H. J. Carmichael, J. Opt. Soc. Am. B 6 (1989) 228.

[7] J. I. Cirac, R. Blatt, A. S. Parkins, and P. Zoller, Phys. Rev. A 49 (1994) 1202.

[8] G. P. Berman, A. M. Iomin, and G. M. Zaslavsky, Physica D 4 (1981) 113; G. P. Berman, E. N. Bulgakov, and D. D. Holm, Cross-over Time in Quantum Boson and Spin Systems (Springer-Verlag, New York, 1994).

[9] See, for instance, Geometric Phases in Physics, ed. A. Shapere and F. Wilczek (World Scientific, Singapore, 1989).

[10] M. V. Berry, J. Phys. A 18 (1985) 15.

[11] J. H. Hannay, J. Phys. A 18 (1985) 221.

[12] C. Jarzynski, Phys. Rev. Lett. 74 (1995) 1264.

[13] A. Perelomov, Generalized Coherent States and Their Applications (Springer-Verlag, Berlin, 1986).

[14] R.Y. Chiao and Y.S. Wu, Phys. Rev. Lett. 57 (1986) 933; A. Tomita and R.Y. Chiao, ibid. 57 (1986) 937; R. Bhandari and J. Samuel, ibid. 60 (1988) 1214; M. Seger, R. Solomon, and Y. Yariv, ibid. 69 (1992) 590. 
[15] P. Hariharan and P.E. Ciddor, Opt. Comm. 110 (1994) 13; P. Hariharan, G. Kieran, G. Larkin, and M. Roy, J. Mod. Opt. 41 (1994) 663; P. Hariharan and M. Roy, ibid. 41 (1994) 2197.

[16] S. Chaturvedi, M.S. Sriram, and V. Srinivasan, J. Phys. A 20 (1987) L1071.

[17] S. Seshadri, S. Lakshmibala, and V. Balakrishnan, Phys. Rev. A 55 (1997) 869.

[18] I. Sh. Averbukh and N. F. Perelman, Phys. Lett. A 139 (1989) 449.

[19] H. P. Yuen, Phys. Rev. A 13 (1976) 2226.

[20] N. Mukunda and R. Simon, Ann. Phys. 228 (1993) 269.

[21] W. de Melo and S. Van Strien, One-Dimensional Dynamics (Springer-Verlag, Berlin, $1993)$.

[22] G. Rauzy, Acta Arithmetica 34 (1979) 315.

[23] N. B. Slater, Proc. Camb. Phil. Soc. 63 (1967) 1115.

[24] As one might expect, the same distribution manifests itself in recurrences to any cell when quasiperiodic flow on a two-torus is coarse-grained. See C. Nicolis, G. Nicolis, V. Balakrishnan, and M. Theunissen, in Stochastic Dynamics, ed. L. SchimanskyGeier and T. Pöschel (Springer-Verlag, Berlin, 1997), pp. 42-54; M. Theunissen, C. Nicolis, and G. Nicolis, J. Stat. Phys. 94 (1999) 437.

[25] P. Shanta, S. Chaturvedi, V. Srinivasan, G. S. Agarwal, and C. L. Mehta, Phys. Rev. Lett. 72 (1994) 1447.

[26] D. L. Aronstein and C. Stroud, Phys. Rev. A 55 (1997) 4526. 